Check for updates

Cite this: RSC Adv., 2017, 7, 35086

Received 1st June 2017

Accepted 14th June 2017

DOI: 10.1039/c7ra06119f

rsc.li/rsc-advances

\section{Tumor targeted nanostructured lipid carrier co- delivering paclitaxel and indocyanine green for laser triggered synergetic therapy of cancer}

\author{
Xuefang Ding, ${ }^{\text {aa }}$ Xian $\mathrm{Xu},{ }^{a}$ Ye Zhao, ${ }^{\mathrm{a}}$ Lihui Zhang, ${ }^{\mathrm{b}}$ Yadong $\mathrm{Yu},{ }^{\mathrm{c}}$ Fei Huang, ${ }^{\mathrm{a}}$ \\ Dezhou Yin ${ }^{a}$ and He Huang (iD) *a
}

A mono approach to cancer therapy usually fails to achieve satisfactory results due to its limited killing effect. Nanostructured lipid carriers (NLCs) with high biocompatibility as well as tumor targetability were successfully designed and formulated in our study to co-deliver paclitaxel (PTX) and indocyanine green (ICG) for combined chemo and photodynamic therapy. ICG not only acted as an imaging reagent but also as a laser responsive agent to aid accelerated drug release under laser irritation. It has been demonstrated that our drug delivery system (DDS) not only significantly enhanced the stability of drugs, produced sufficient local ROS and triggered accelerated drug release upon laser irradiation, but also increased intracellular uptake of drugs and induced increased cytotoxicity in cancer cells via synergistic effects. Moreover, NLCs demonstrated excellent tumor targetability in tumor-bearing mice. Therefore, tumor targeted co-delivery of PTX and ICG mediated by NLCs could be a promising approach for further development as an effective strategy for cancer therapy.

\section{Introduction}

To date, successful cancer therapy remains a major challenge to cure cancer. ${ }^{1}$ Chemotherapy using paclitaxel (PTX) as an anticancer drug has been applied in many clinical trials which showed positive results in extending the lifetime of recipients. ${ }^{2,3}$ However, traditional chemotherapy, although widely adopted, has multiple disadvantages such as unwanted side effects and multidrug resistance which strongly compromise the final outcome. ${ }^{4}$

As a newly investigated category of approach for medicinal purposes, photodynamic therapy (PDT) offers a new way to damage cancer cells by generating highly reactive oxygen species (ROS), such as singlet oxygen $\left({ }^{1} \mathrm{O}_{2}\right)$, in response to light irritations, ${ }^{5}$ which can achieve controlled release or effect. ${ }^{6}$ Indocyanine green (ICG) as the only PDT agent approved by the FDA for medical imaging and diagnosis in clinic, has advantages over other competitors on safety. However, several major limitations, including irreversible rapid degradation in aqueous solution, concentration dependent aggregation, short blood half-life (2-4 min), lack of targeting capacity and risk of photo

${ }^{a}$ School of Pharmaceutical Science, Nanjing Tech University, No. 30 South Puzhu Road, Nanjing,211816, China.E-mail:dxf0118@njtech.edu.cn; biotech@njtech.edu.cn ${ }^{b}$ Jiangsu National Synergetic Innovation Center for Advanced Materials (SICAM), Nanjing Tech University, No. 30 South Puzhu Road, Nanjing, 211816, China

${ }^{c}$ College of Biotechnology and Pharmaceutical Engineering, Nanjing Tech University, No. 30 South Puzhu Road, Nanjing 211816, China bleaching, strictly limit its further application on cancer therapy that requires the aid of additional drug delivery systems (DDS). ${ }^{7}$

Nanostructured lipid carrier (NLC) is the second generation of solid lipid drug carriers which was nano-scaled particles automatically formed in aqueous solutions. ${ }^{8}$ As a new type of delivery system, NLC protected drugs usually show improved performance in terms of long-term stability and bioavailability. ${ }^{9}$ NLC is composed of both solid lipids and liquid lipids (oils) that originated from natural materials. ${ }^{10}$ Due to its highly unordered lipid structures, NLC offers the possibility to accommodate a variety of hydrophobic drugs, including doxorubicin and PTX. ${ }^{8,11}$ Despite its biocompatible nature, NLC can also be easily surface modified with functional segments, including stealthy polymers and tumor targeting ligands. ${ }^{12}$

So far, it has been demonstrated that mono therapy strategies, either chemotherapy or PDT, has its limitations. ${ }^{13}$ In order to employ the advantages of their merits while avoiding their shortcomings, combination therapy using different drugs is emerging as a preferable anti-cancer approach due to its enhanced efficacy when compared with mono therapy strategies. ${ }^{14,15}$ Here in our study, tumor-targeted NLC was firstly fabricated using polyethylene glycol (PEG) and folic acid (FA) as the shielding and tumor-homing segments. During this process, ICG and PTX were incorporated into the hydrophobic lipid core of the NLC to formulate a co-delivery system. ICG not only acted as a therapeutic drug but also as a laser responsive agent to aid accelerated PTX release under laser irritation. It was expected that the as-prepared NLC can escape the reticuloendothelial system capture, selectively accumulated to the tumor 
tissue and response to laser irritation which result in enhanced anti-cancer efficacy.

\section{Materials and methods}

\subsection{Materials}

Glycerin monostearate (GM), oleic acid (OA) and paclitaxel (PTX) were purchased from Sigma-Aldrich (St Louis, MO, USA). Folic acid (FA), Hoechst 33342, ICG, 2',7'-dichlorfluoresceindiacetate (DCFH-DA), methylthiazoletetrazolium (MTT) and 1,3-diphenylisobenzofuran (DPBF) were offered by Thermo Fisher Scientific (Massachusetts, USA). Stearic acid-polyethylene glycol and stearic acid-polyethylene glycol-folate (SAPEG-FA) was purchased from Nanocs Inc. (New York, USA). Other chemicals involved in the study were analytically pure obtained from Shanghai Chemical Reagent Co. Ltd., (Shanghai, China) and used without further pretreatment.

\subsection{Cell culture}

Human liver carcinoma cell line (HepG2) and mouse fibrosis cell line (NIH3T3) purchased from the Cell Bank of Shanghai Institute of Biochemistry and Cell Biology, Chinese Academy of Sciences (Shanghai, China) were cultured in DMEM medium containing $10 \%$ fetal bovine serum (FBS), $100 \mathrm{U} \mathrm{mL}^{-1}$ of penicillin and $100 \mu \mathrm{g} \mathrm{mL} \mathrm{m}^{-1}$ of streptomycin (Gibco, USA) at $37{ }^{\circ} \mathrm{C}$ using a humidified $5 \% \mathrm{CO}_{2}$ incubator (311, Thermo scientific, USA).

\subsection{Animal model}

Male BALB/c nude mice and New Zealand rabbits were purchased from Shanghai Lab. Animal Research Center. All animal experiments were conducted in strict compliance with the National Institute of Health Guide for the Care and Use of Laboratory Animals and approved by Animal Ethics Committee of Nanjing Tech University. In order to obtain xenograft HepG2 tumor xenograft mice, approximately $10^{7}$ of HepG2 cells were incubated subcutaneously to the flank region of the mice. Tumor volume $(V)$ was determined by measuring length $(L)$ and width $(W)$, and calculated as formula: $V=L \times W 2 / 2$.

\subsection{Preparation of NLC}

GM (7 mg), OA (4 mg), SA-PEG-FA ( $3 \mathrm{mg})$ and SA-PEG-FA (2 $\mathrm{mg}$ ) were added into $1 \mathrm{~mL}$ of ethanol, and heated in water bath $\left(70{ }^{\circ} \mathrm{C}\right)$ to obtain a transparent solution. To a preheated $\left(70{ }^{\circ} \mathrm{C}\right)$ aqueous solution $(10 \mathrm{~mL})$ containing $0.3 \%$ poloxamer $188(\mathrm{w} / \mathrm{v})$, the mixture of lipid components was injected constantly via syringe under gentle agitation. The aqueous solution was allowed to agitate for another $10 \mathrm{~min}$ after the addition of lipids. Afterwards, the solution was stored in $4{ }^{\circ} \mathrm{C}$ for further use.

The drug (PTX and/or ICG) was loaded into NLC by physical encapsulation. Drug was dissolved with lipids in the organic phase, and the synthesis proceeded as described above. After that, the solution was centrifuged at $1000 \mathrm{rpm}$ for $30 \mathrm{~min}$ (Allegra 64R, Beckman Coulter, USA), followed by dialysis against deionized water for $4 \mathrm{~h}$ (MWCO: $3.5 \mathrm{kDa}, 2 \mathrm{~L} \times 4$ ) to remove the unloaded drugs.

\subsection{Characterization of NLC}

The particle size of NLC was measured by Zetasizer Nano ZS90 (Malvern Instruments Ltd., UK). The shape and surface morphology of NLC were observed by transmission electron microscopy (TEM, JEOL 100CX II, Tokyo, Japan).

\subsection{Stability, hemolysis and drug release}

For colloidal stability test, the freshly prepared NLC was diluted with phosphate buffer (PBS, pH 7.4) at the volume ratio of $1: 10$. The change in particle size was recorded using Zetasizer Nano ZS90 at predetermined time intervals (2, 4, 6, 8, 10, 12 and $24 \mathrm{~h})$ for up to $24 \mathrm{~h}$. To evaluate photostability of ICG loaded NLC, fluorescence signal intensity was determined using a fluorescence spectrometer (Hitachi F-7000, Japan) with excitation wavelength of $740 \mathrm{~nm}$. Free ICG and ICG loaded NLC $(1 \times$ $10^{-4} \mathrm{M}$ of ICG) were excited with a $150 \mathrm{~W}$ xenon lamp continuously for $1 \mathrm{~h}$ and the fluorescence intensity at different time points $(10,20,30,40,50$ and $60 \mathrm{~min})$ was recorded. Dialysis method was adopted to assess the in vitro release profile of PTX and ICG from different formulations. Tested samples were placed into a dialysis bag (MWCO $3.5 \mathrm{kDa}$ ), immersed in PBS containing $0.1 \%$ Tween 80 at $37^{\circ} \mathrm{C}$ with or without $808 \mathrm{~nm}$ laser irradiation $\left(1 \mathrm{~W} \mathrm{~cm}^{-2}, 5 \mathrm{~min}\right)$, and agitated at $100 \mathrm{rpm}$ using a shaking water bath. At pre-determined time points, five milliliter of the dialysate was taken out while the same amount of fresh media was added back and kept in the water bath for further study. The collected primary medium was then subjected to UV analysis at $227 \mathrm{~nm}$ and $805 \mathrm{~nm}$ for PTX and ICG content, respectively. For hemolysis assay, Red blood cells (RBCs) were firstly obtained from New Zealand rabbit and diluted to $2 \%$ suspension with saline solution. Then the RBCs were formulated as a $2 \%$ suspension with saline solution. NLC were added into $2 \%$ RBCs suspension with same volume to achieve the designated concentrations $(0.1,0.25,0.5,0.75,1$, $1.25,1.5,1.75$ and $2 \mathrm{mg} \mathrm{mL}^{-1}$ ) and incubated at $37^{\circ} \mathrm{C}$ for $1 \mathrm{~h}$. Besides, RBCs suspension was also incubated with saline and distilled water under the same condition as negative $(0 \%$ hemolysis) and positive controls ( $100 \%$ hemolysis), respectively. After that, all the samples were centrifuged at $3000 \mathrm{rpm}$ for 10 min and the absorption values of same volume of supernatants which represented the counts of released hemoglobin were measured by ultraviolet (UV) spectrophotometer (TU-1810, Purkinje, China).

\subsection{In vitro $\mathrm{ROS}$ generation detection}

The generation of singlet oxygen was detected by DPBF as reported in previous article. ${ }^{16}$ In a typical experiment, $20 \mu \mathrm{L}$ DPBF solution (DMSO, $10 \mathrm{mM}$ ) was added to drug loaded NLC (ICG concentration: $0.1,0.2,0.3,0.4$ and $0.5 \mathrm{mg} \mathrm{mL}^{-1}$ ). The mixture was irradiated with a laser bean at $1 \mathrm{~W} \mathrm{~cm}^{-2}$. At predetermined time intervals, the absorption spectra of DPBF were recorded at points by a UV spectrophotometer and the values of absorption at $415 \mathrm{~nm}$ were plotted against irradiation time or concentration. 


\subsection{In vitro cellular uptake}

HepG2 cells were seeded into 24-well plates at the cell density of $3 \times 10^{4}$ cells per well and allowed to culture overnight. After that, the primary medium was replaced with fresh serum-free medium containing drug loaded NLC (ICG concentration: $0.1 \mathrm{mg} \mathrm{mL}^{-1}$ ). For competitive binding assay, excessive of FA (a final concentration of $1 \mathrm{mg} \mathrm{mL}^{-1}$ ) was added $2 \mathrm{~h}$ prior to NLC. At pre-determined time interval, cells were washed thoroughly with cold PBS, fixed by $4 \%$ formalin, followed by Hoechst 33242 staining $\left(10 \mu \mathrm{g} \mathrm{mL} \mathrm{m}^{-1}, 15 \mathrm{~min}\right)$ and then subjected to inverted fluorescence microscope observation (Axio Observer A1, Zeiss, Germany). On the other hand, cells were harvested after trypsinization, resuspended in PBS and subjected to flow cytometer for qualitative fluorescence analysis (FC500MCL, Beckman Coulter, USA).

\subsection{Intracellular ROS detection}

DCFH-DA is the derivative of DCF which remains nonfluorescent at its reduced state and can be taken into cells via endocytosis. Once being exposed to ROS, DCFH-DA can be oxidized into DCF and becomes highly fluorescent. HepG2 cells were seeded in 24-wells as described above. Following incubation of different formulations (ICG concentration: $0.1 \mathrm{mg} \mathrm{mL}{ }^{-1}$ ) for $4 \mathrm{~h}$, cells were washed and treated with $25 \mu \mathrm{M}$ of DCFH-DA at $37^{\circ} \mathrm{C}$ in dark for $30 \mathrm{~min}$. Cells were then washed thoroughly with cold PBS and exposed to $808 \mathrm{~nm}$ irradiation for $5 \mathrm{~min}(1 \mathrm{~W}$ $\mathrm{cm}^{-2}$ ). After irradiation, cells were subjected to fluorescence microscope observation.

\subsection{In vivo imaging and biodistribution}

When tumor volume reaches around $100 \mathrm{~mm}^{3}$, the tumorbearing mice were randomly divided into three groups $(n=$ 3). $200 \mu \mathrm{L}$ of free ICG and drug loaded NLC were individually injected into the mouse via tail vein administration $(20 \mu \mathrm{g}$ ICG per mouse). Images were taken at 2, 4 and $8 \mathrm{~h}$ after injection using the in vivo imaging system (FX PRO, Kodak, USA). After in vivo imaging, the mice were sacrificed and their main organs (hearts, livers, spleens, lungs, kidneys) as well as tumors were excised to measure their individual fluorescence intensities.

\subsection{In vitro cytotoxicity assays}

HepG2 cells $\left(3 \times 10^{3}\right.$ cells per well $)$ were seeded in 96-well plates and cultured overnight. The culture medium was replaced with $200 \mu \mathrm{L}$ of serum-free medium containing Taxol, PTX loaded NLC, ICG loaded NLC and co-loaded (PTX + ICG) NLC at different drug concentrations for $48 \mathrm{~h}$ with irradiation treatment $\left(1 \mathrm{~W} \mathrm{~cm}^{-2}\right)$ for $5 \mathrm{~min}$. Afterwards, the medium was replaced with an equal volume of fresh medium containing $5 \mathrm{mg} \mathrm{mL}{ }^{-1} \mathrm{MTT}$ and incubated for $4 \mathrm{~h}$ at $37^{\circ} \mathrm{C}$. Then MTT was removed, and the formazan was dissolved with dimethyl sulfoxide (DMSO) with stirring for 15 min on a microtiter plate shaker. The cell viability was estimated according to the absorption values determined by a microplate reader (Bio-Rad, model 680, USA) at the wave length of $570 \mathrm{~nm}$.

To establish the HepG2 spheroids, HepG2 and NIH3T3 cells were detached from monolayers, pipetted into single cell suspensions $\left(1.5 \times 10^{4}\right.$ cells per $\left.\mathrm{mL}\right)$ and mixed with each other (v/v, $1: 1)$. Afterwards, cells were transferred into flat-bottomed 96-well plates precoated with $2 \%$ agarose $(200 \mu \mathrm{L}$ per well). Cells were incubated for approximately 4 days, formulations were added into the spheroids by partially $(100 \mu \mathrm{L})$ replace the culture medium with fresh medium diluted formulations. The drug concentration was fixed at $1 \mu \mathrm{g} \mathrm{mL}^{-1}$. The formulations were allowed to culture with spheroids for $12 \mathrm{~h}$ and all spheroids were treated with $808 \mathrm{~nm}$ irradiation $\left(1 \mathrm{~W} \mathrm{~cm}^{-2}\right)$ for $5 \mathrm{~min}$. The change in spheroids was monitored by inverted microscope every day for 4 days.

\section{Results and discussion}

\subsection{Preparation and characterization of NLC}

A solvent diffusion method was employed in our study to prepare the NLC. The drug loading process was accompanied with the preparation of the NLC. During the diffusion of lipid components from organic phase into aqueous medium, the lipid components can self-assembled into well-dispersed nanoscaled particles. In our study, the polymer SA-PEG-FA containing stealthy polymer PEG as well as tumor targeting ligand FA was adopted to form a shielding and tumor targeting layer on the surface of the as-prepared NLC. The amphiphilic ICG
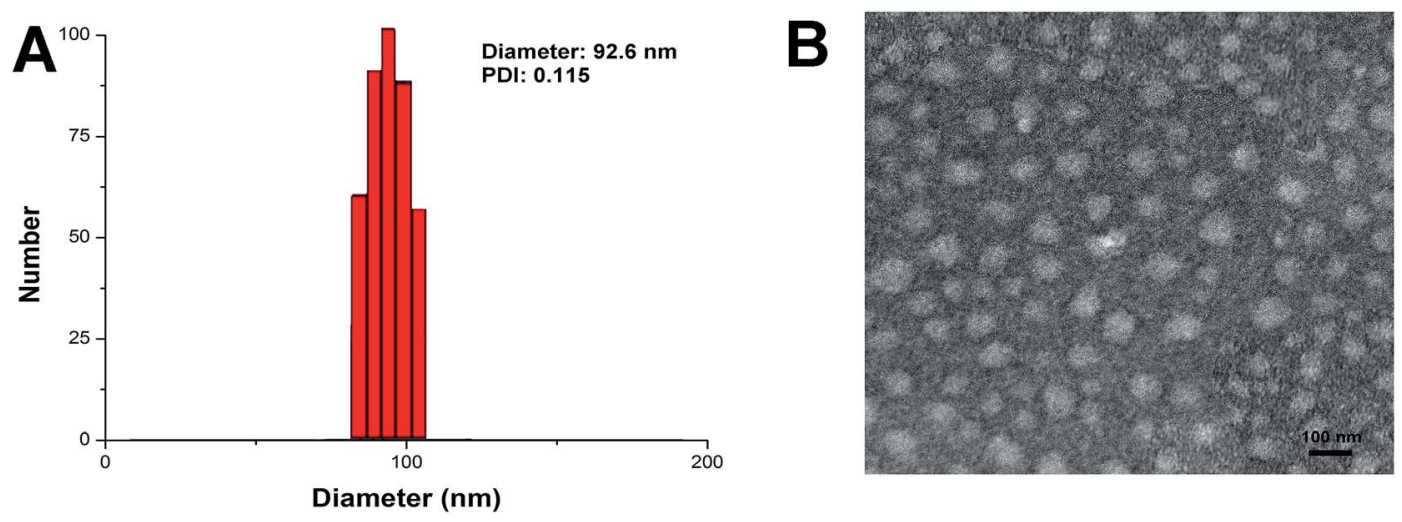

Fig. 1 (A) Particle size distribution of NLC. (B) TEM image of NLC. Scale bar: $100 \mathrm{~nm}$. 

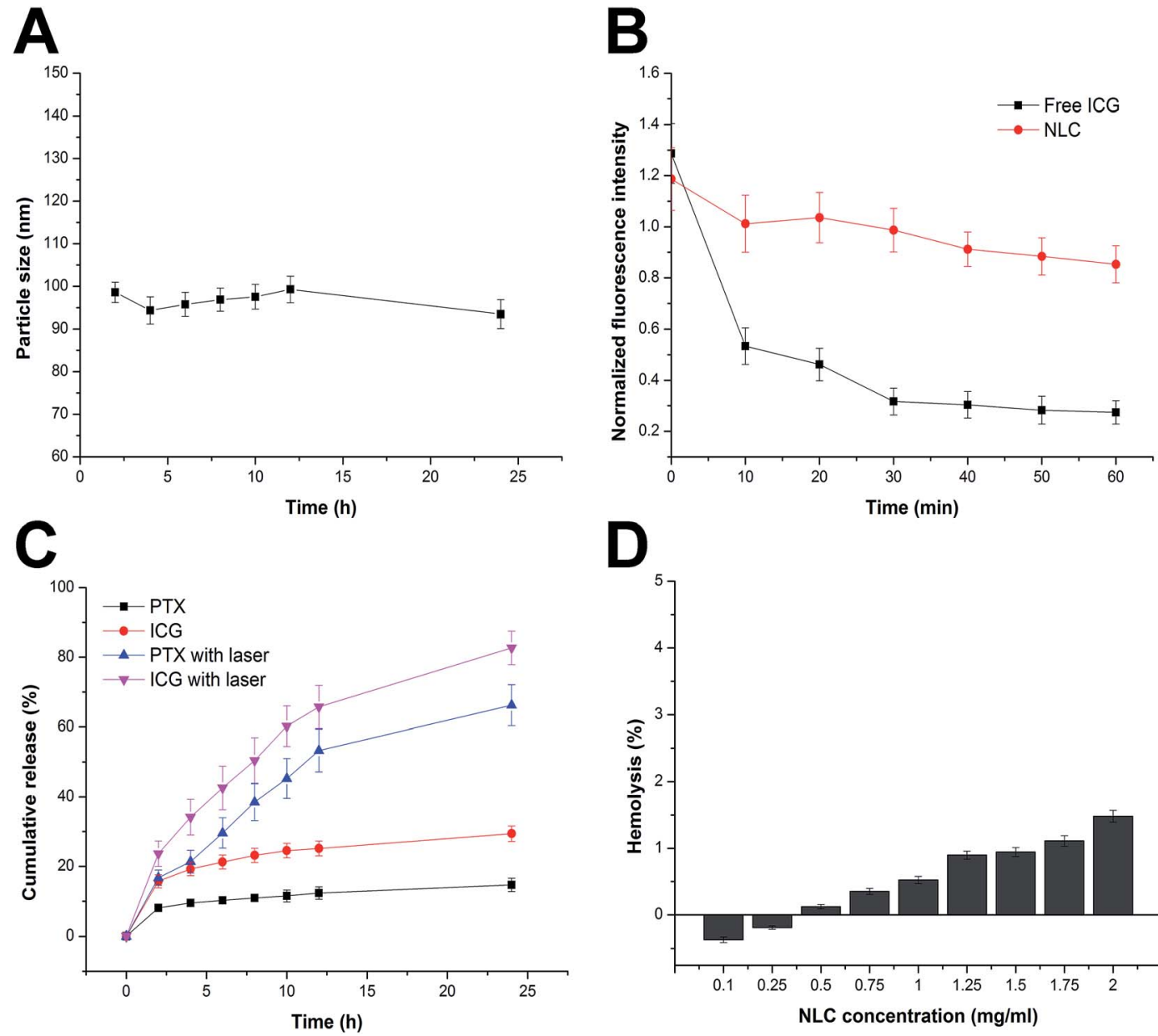

D

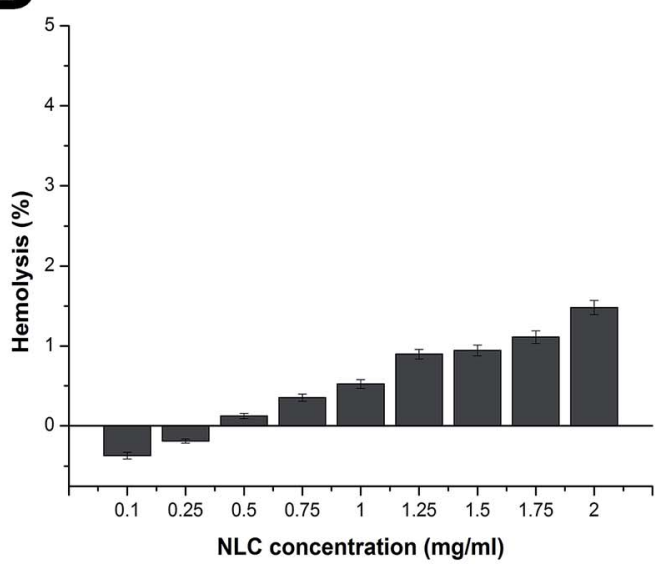

Fig. 2 (A) Colloidal stability of NLC in PBS (pH 7.4) at $37^{\circ} \mathrm{C}$ for up to $24 \mathrm{~h}$. (B) Fluorescent stability of ICG in different nanoparticles under $1 \mathrm{~h}$ exposure of a $150 \mathrm{~W}$ xenon lamp. (C) Drug (PTX and ICG) release profiles of NLC in PBS (pH 7.4) at $37^{\circ} \mathrm{C}$ with or without laser irritation. (D) Hemolysis of NLC at various concentrations. Data were shown as mean \pm S.D. $(n=3)$.
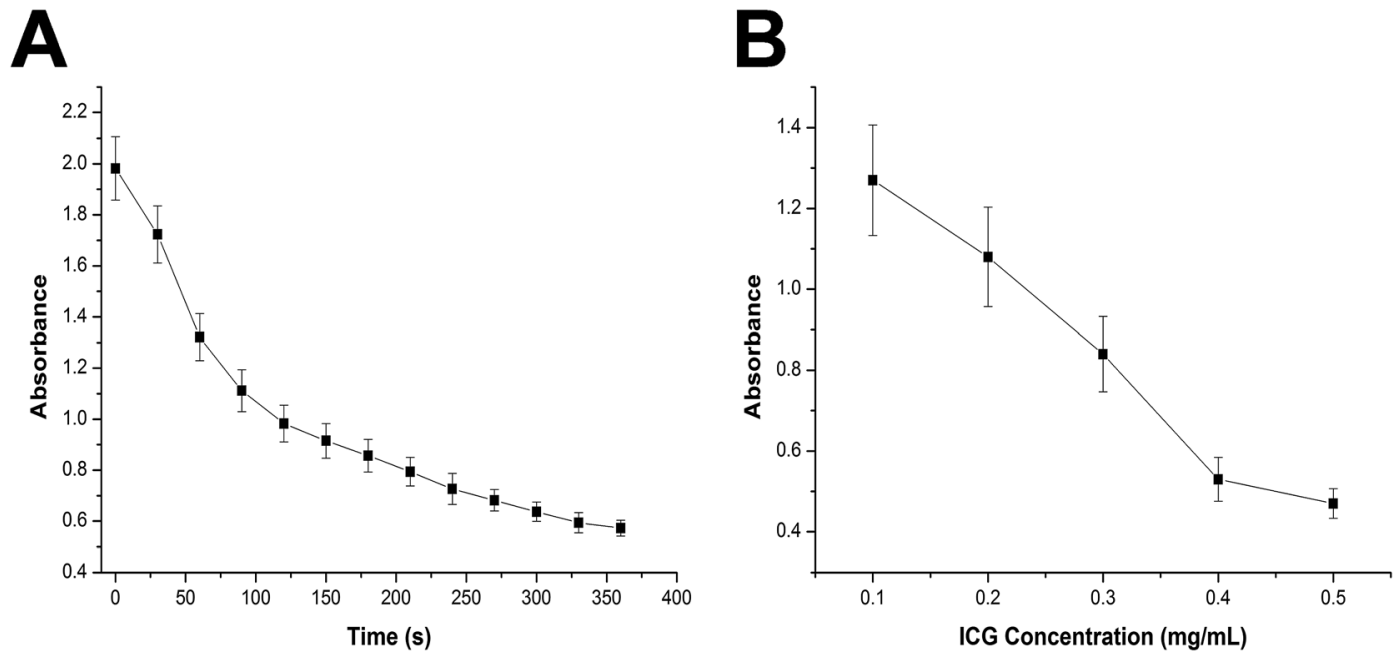

Fig. 3 (A) Variation on absorbance of DPBF in the presence of drug loaded NLC (ICG concentration $0.1 \mathrm{mg} \mathrm{mL}^{-1}$ ) after irradiation (1 W $\mathrm{cm}^{-2}$ ) for different time intervals. (B) Variation on absorbance of DPBF in the presence of different concentrations of drug loaded NLC (ICG concentration: $\left.0.1-0.5 \mathrm{mg} \mathrm{mL}^{-1}\right)$ under irradiation for $60 \mathrm{~s}$. Data were shown as mean \pm S.D. $(n=3)$. 
and hydrophobic PTX was encapsulated in the lipid matrix of NLC in the same solvent diffusion process. After drug loading, the resulted NLC was subjected to size measurement using dynamic light scattering (DLS) method. As shown in Fig. 1A, the hydrodynamic diameter of blank NLC was determined as $92.6 \mathrm{~nm}$ with a polydispersion index (PDI) value of 0.115 . Drug loading of NLC did not significantly change its particle size and PDI value (data not shown). Morphology observation using TEM (Fig. 1B) further revealed that NLC was near spherical nanoparticles which dispersed homogeneously on the copper screen with a diameter of around $100 \mathrm{~nm}$. These results are highly accords to that obtained by DLS. It has been demonstrated by
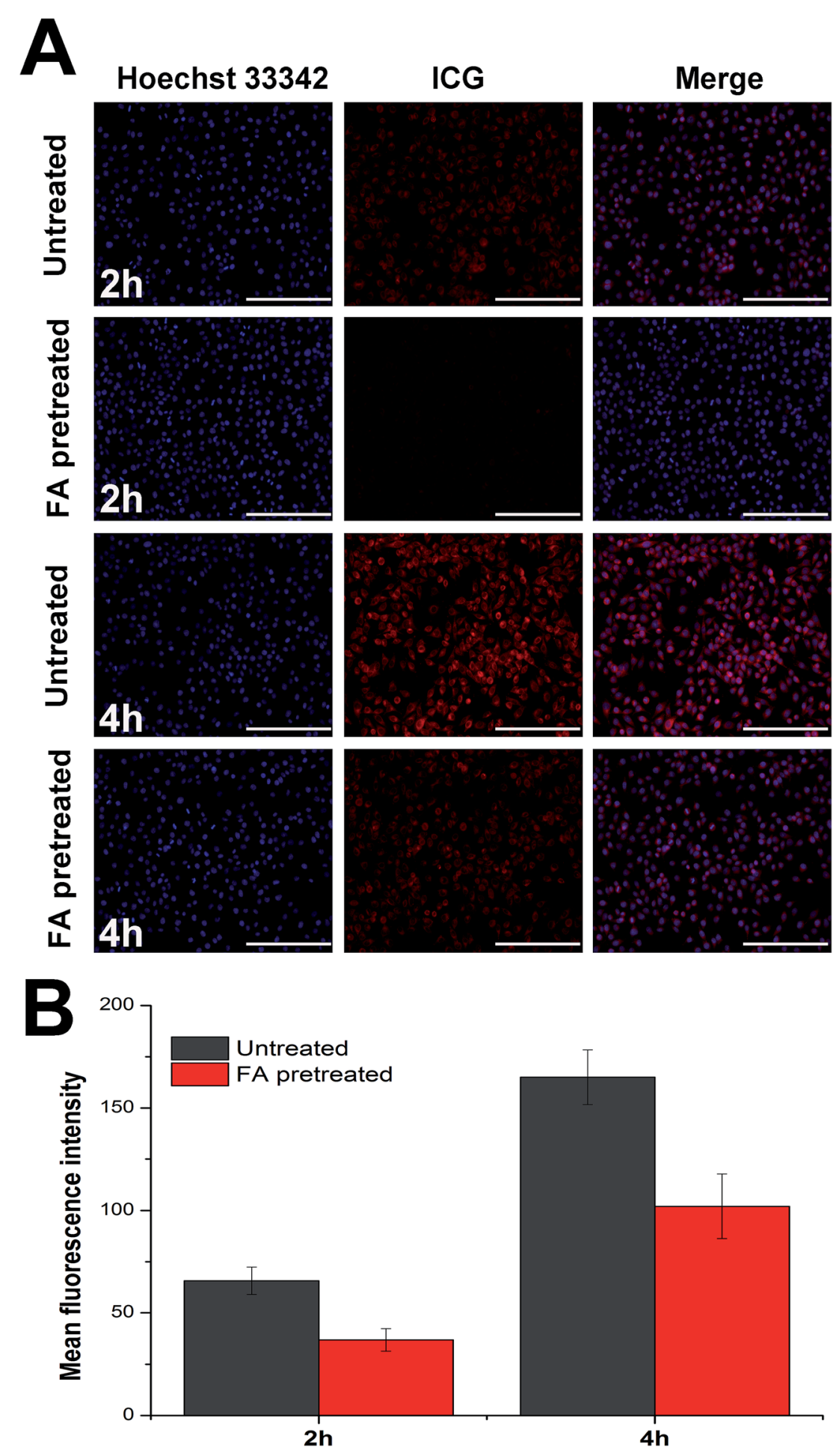

Fig. 4 The in vitro cellular uptake analysis of NLC in HepG2 cells. (A) Inverted fluorescence microscope images of NLC treated cells with or without FA pretreatment at 2 and $4 \mathrm{~h}$ post-incubation. Scale bar: $500 \mu \mathrm{m}$. (B) Flow cytometric analysis of MFI in cells treated by NLC with or without FA pretreatment for 2 and 4 h. Data were shown as mean \pm S.D. $(n=3)$. 
previous reports ${ }^{17,18}$ that particles with size under $200 \mathrm{~nm}$ can effectively take advantage of the high permeability of tumor tissue to target and accumulate at the tumor site (also known as enhanced permeability and retention effect, EPR). On the other hand, surface modification with stealthy PEG polymer was generally considered as a potent way to avoid interactions with anionic plasma components within the circulation system, ${ }^{16}$ which was beneficial for nanoparticles to escape the reticuloendothelial system (RES) capture and prolong the circulation time.

\subsection{Stability, hemolysis and drug release}

In order to safely deliver the encapsulated cargos to the targeted site. There are some basic requirements for an ideal drug delivery system. Firstly, it should be able to protect the entrapped therapeutic cargos under physiological conditions without significant drug leakage and size change for a period of time (usually $24 \mathrm{~h}$ ). ${ }^{19}$ Secondly, the release of cargos can be controlled after reaching the site of action. ${ }^{20}$ At last but not least, the drug delivery system should not induce any adverse reaction, such as hemolysis, during the whole process. ${ }^{2}$ In our study, drug loaded NLC was investigated systematically on their colloidal stability, hemolysis and controlled release. To estimate the colloidal stability of the described nanoparticle, the change in particle size of drug-loaded NLC, which serves as an indicator of size change under physiological conditions, was monitored in PBS (pH 7.4) for 24 h. As shown in Fig. 2A, the size of drug-loaded NLC remained stable during the whole period with only slight fluctuation which indicated that the asprepared delivery system was capable of maintaining its size without being affected by the environment for a long time. This is beneficial for its further development as a stable platform with predictable behavior to fit more extensive applications.

During the delivery process, the encapsulated drug was supposed to be well protected by the delivery system. ICG is a FDA-approved theranostic agent which is sensitive to light and heat once being dispersed in aqueous solution. As we all know that its optical properties may greatly affect the efficiency of PDT treatments, the photostability of drug loaded NLC was worth investigating. The fluorescent intensity of encapsulated ICG was shown in Fig. 2B. In comparison with free ICG, fluorescent intensity of drug loaded NLC was more stable under continuous illumination. The fluorescent intensity of drug loaded NLC decreased to $71.86 \%$, while the free ICG dropped to $21.29 \%$. These results demonstrated that the stability of ICG was significantly improved after encapsulation by NLC.

After reaching the targeted sites, the drug release of the delivery system was expected to accelerate. Optimal biological activity usually requires strictly controlled drug release of the applied delivery system. The release profile of both drugs (PTX and ICG) was evaluated in PBS (pH 7.4) at $37^{\circ} \mathrm{C}$ with or without laser irradiation. As shown in Fig. 2C, both PTX and ICG were released from NLC at a relatively slow profile $(14.71 \%$ and $29.43 \%$, respectively, at $24 \mathrm{~h}$ of incubation) without laser irradiation, which indicated that the NLC can restrain the drug from leakage within physiological conditions for a long time. However, it was interesting to find that upon laser irradiation, the release rate of both PTX and ICG from NLC increased rapidly (66.62\% and $82.67 \%$, respectively, at $24 \mathrm{~h}$ of incubation). These results indicated the rate of the release could be accelerated once triggered by light irradiation. It was inferred that upon laser irritation, ICG absorbed the energy, become highly active and was capable of generating singlet oxygen. On the other hand, as a widely adopted theranostic agent, ICG could also generate heat upon laser irritation. Both ways can compromise the stability of NLC, destroy the intact structure of the nanoparticle that finally lead to burst release of the encapsulated drugs. ${ }^{21}$

Apart from steadily delivering and controlled release properties, hemolysis of the drug loaded NLC was also investigated as a safety guide for intravenous administration and medicinal application. As shown in Fig. 2D, drug loaded NLC displayed no
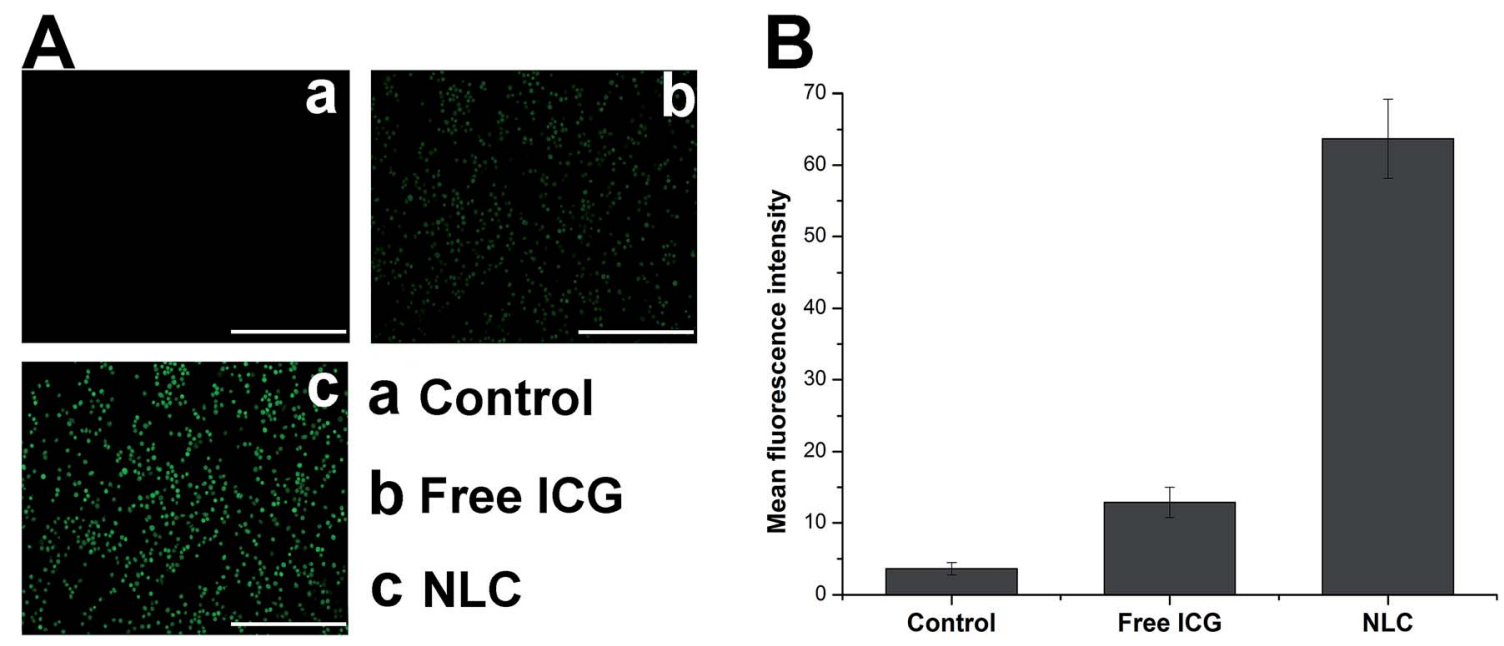

Fig. 5 The in vitro ROS generation analysis and cytotoxicity of NLC in HepG2 cells. (A) Representative inverted fluorescence microscope images of cells treated with free ICG and drug-loaded NLC after 5 min laser irradiation. Scale bar: $500 \mu \mathrm{m}$. (B) Flow cytometric analysis of MFI in cells treated by free ICG and drug-loaded NLC. Data were shown as mean \pm S.D. $(n=3)$. 
or neglectable hemolysis (merely $1.48 \%$ ) on RBCs at the highest concentration of $2 \mathrm{mg} \mathrm{mL}{ }^{-1}$, suggesting that the as-prepared formulation exhibited great hemocompatibility. This could be explained by the fact that NLC was composed by naturally derived materials. In all, it has been demonstrated that drug loaded NLC exerted a stable and controlled-release capability with high biocompatibility which holds great promise to benefit therapeutic effect in vivo.

\subsection{ROS detection}

Whether the encapsulated ICG still maintain the capability to generate ROS is prerequisite for drug loaded NLC to serve as a PDT system. As a result, the generation of ROS by drug loaded NLC was detected by DPBF. It has reported by previous report that DPBF would quench its intrinsic absorbance at $415 \mathrm{~nm}$ via forming DPBF-endoperoxide complex with environmental ROS. The decrease of the absorbance of DPBF in solution over time upon irradiation was detected to evaluate the efficiency of ROS production. As displayed in Fig. 3A, the absorbance of DPBF in the presence of drug loaded NLC (ICG concentration $0.1 \mathrm{mg}$ $\mathrm{mL}^{-1}$ ) after irradiation various as the laser irritation continues. In detail, intensity of DPBF at $415 \mathrm{~nm}$ displayed a continuous decrease which achieving almost $71 \%$ absorbance quench at $360 \mathrm{~s}$ post irritation. The absorbance variation of DPBF at $415 \mathrm{~nm}$ as a function of ICG concentration was further investigated. Different concentrations of drug loaded NLC (ICG concentration: $0.1-0.5 \mathrm{mg} \mathrm{mL}^{-1}$ ) under the same irradiation condition ( $1 \mathrm{~W} \mathrm{~cm}^{-2}$ for $60 \mathrm{~s}$ ) was employed. It was shown in Fig. 3B that drug loaded NLC exhibited a concentrationdependent production of ROS in aqueous solution upon laser irradiation. These results confirmed the fact that the encapsulated ICG within NLC still preserve the capability to generate ROS.

\subsection{In vitro cellular uptake}

Cell membrane is composed of lipid bilayer which selectively allowing chemicals in and out of the cell depending on the size, molecular weight and polarity. It has been generally recognize that lipid materials which have similar properties to the lipid bilayer, are more likely to be taken up by cells. The general components of NLC are physiological lipids which are supposed to have high affinity to the lipid bilayer. On the other hand, the FA ligands exposed toward the surface of the nanoparticles can target FA receptor which excessively expressed in tumor cells. Herein, we speculated that NLC wound provide an activetargeting platform for the delivery of ICG. In vitro cell imaging was conducted to explorer the cellular uptake profile of NLC. HepG2 cells were incubated with drug loaded NLC at the ICG concentration of $0.1 \mathrm{mg} \mathrm{mL}{ }^{-1}$ for different time intervals $(2 \mathrm{~h}$ and $4 \mathrm{~h}$ ). Internalization of drug loaded NLC was visualized by inverted fluorescence microscope. As shown in Fig. 4A, ICG showed strong red fluorescent signal in cells treated with drug loaded NLC. The fluorescent signal increased as a function of time which indicated that the cellular uptake process was positively related to the incubation time. It was worth mentioning that when the cells were pretreated with a high dose of free FA to competitively block the FA receptors, the intracellular fluorescent signal of ICG was significantly dropped to a lower level, indicating that the cellular uptake of NLC is largely dependent on receptor mediated internalization. To further study the cellular uptake efficiency, the mean fluorescent intensity (MFI) of cells incubated for different time intervals was quantitatively determined using flow cytometry (Fig. 4B). The results obtained by flow cytometry were in great accordance with that observed using fluorescence microscope. These results both confirmed that NLC facilitated augmented internalization in tumor cells via receptor-mediated pathway. Thus,
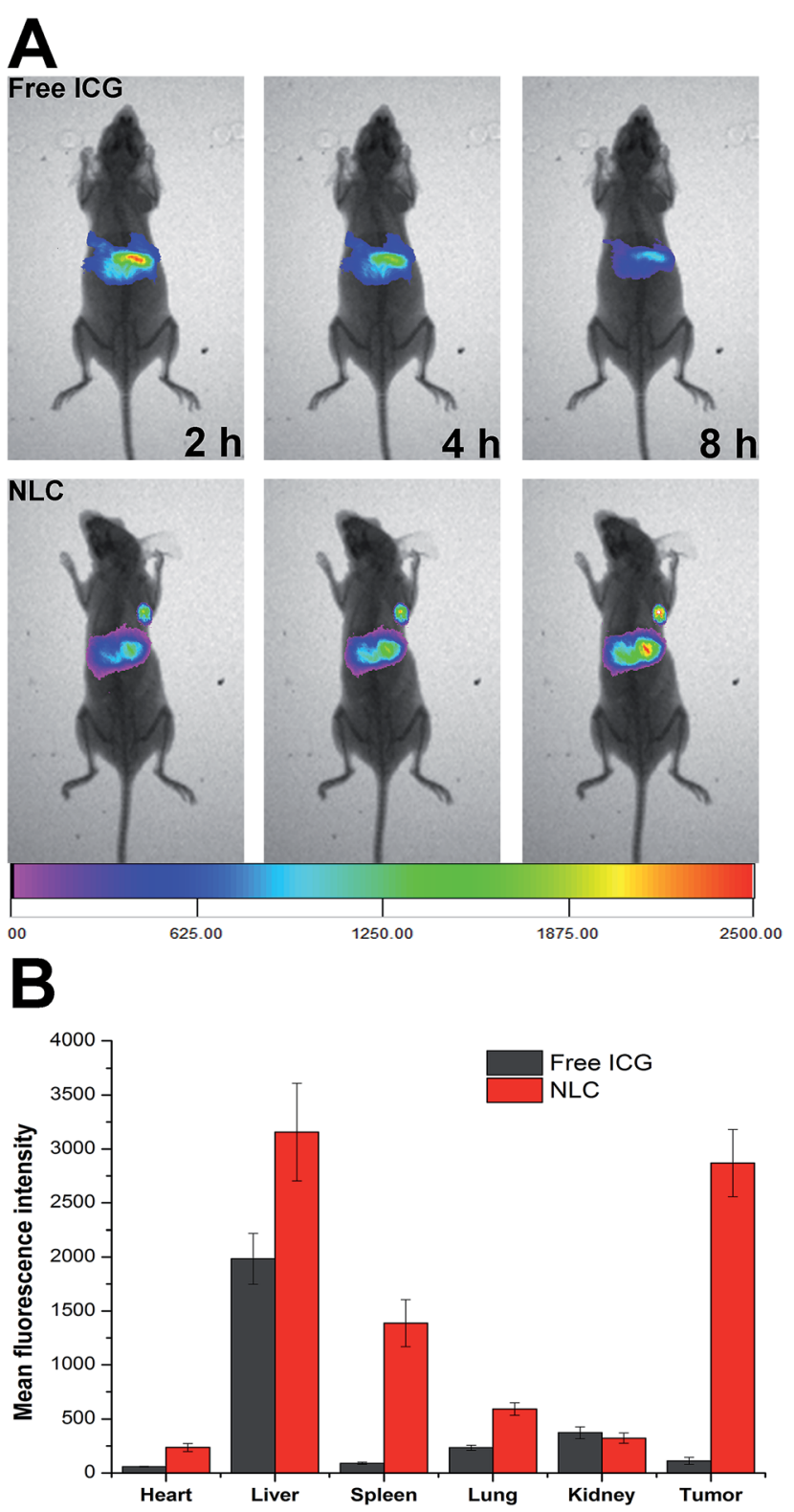

Fig. 6 In vivo imaging and biodistribution of nude mice bearing HepG2 tumors after injection of ICG-loaded nanoparticles. (A) Timelapse NIR fluorescence images of mice after intravenous injection of free ICG and drug-loaded NLC. (B) Semiquantitative analysis of biodistribution of free ICG and drug-loaded NLC in mice determined by MFI in the tumor or tissues. Data were shown as mean \pm S.D. $(n=3)$. 
NLC functions as targeted transporters that provide new possibilities for enhanced PDT effect.

\subsection{Intracellular ROS detection}

The generation of ROS is the major cause for the cancer cell apoptosis of PDT. As a result, it is important to verify whether the drug loaded NLC still capable of generating significant amount of ROS within cancer cells upon laser irritation. DCFHDA as a fluorescence probe was employed here to detect the generated ROS within cancer cells. As a sensitive indicator of intracellular ROS, non-fluorescent DCFH-DA enters into can reacts with ROS after being uptaken into cells to produce its oxidized product DCF, an with green fluorescence that can be detected at Ex $485 \mathrm{~nm} / \mathrm{Em} 530 \mathrm{~nm}$. As shown in Fig. 5A, after irradiation ( $808 \mathrm{~nm}, 5 \mathrm{~min}$ ), almost no fluorescent signal was detected in blank control group (without adding ICG), as ROS generation is these cells was faint. Observable weak fluorescent signal was found in cells treated with free ICG. In contrast, fluorescent intensity of DCF in HepG2 cells treated with NLC increased substantially compared with free ICG and control group, suggesting effective ROS production. MFI obtained by flow cytometry (Fig. 5B) also confirmed these results. Besides, it was also noticed that the fluorescent signal was homogeneously in both cytoplasm and nucleus, implying a broad distribution of ROS. This result was also consistent with the diffuse pattern displayed in cellular uptake experiment. Therefore, NLC is considered to be a suitable carrier to achieve great PDT effect via efficient delivery of ICG to intracellular site.

\subsection{In vivo imaging and biodistribution}

To study the in vivo fate of NLC, biodistribution of drug loaded NLC in compare to free ICG in HepG2 tumor-bearing mice was monitored by a non-invasive imaging approach with ICG as a near infrared fluorescence probe. As displayed in Fig. 6A, the majority of the fluorescent signals of free ICG were found in the liver within $2 \mathrm{~h}$ after injection. On the other hand, fluorescent signals decreased as the increase of injection time, which indicated that free ICG has a short circulation within the body. Rapid aggregation of free ICG in physiological environments

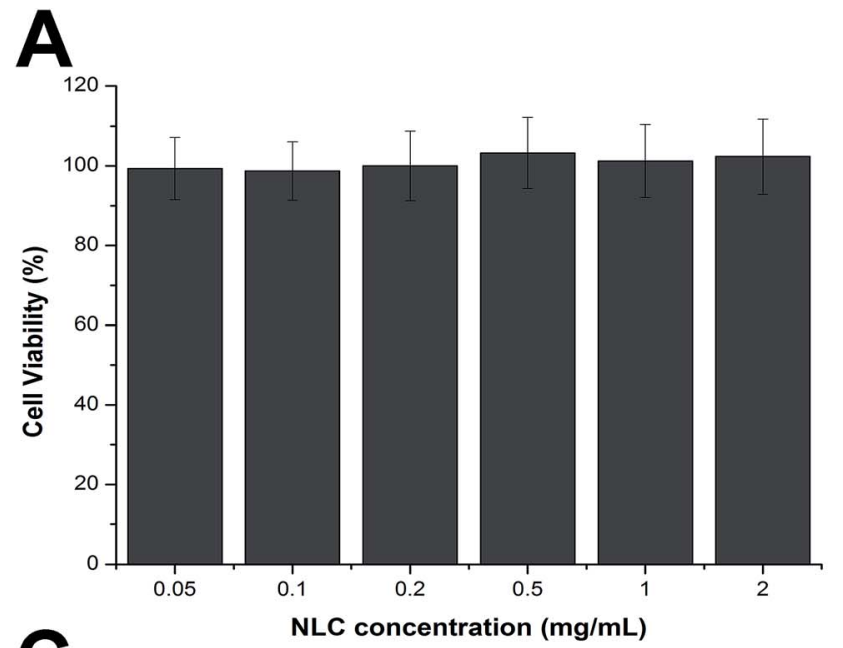

B
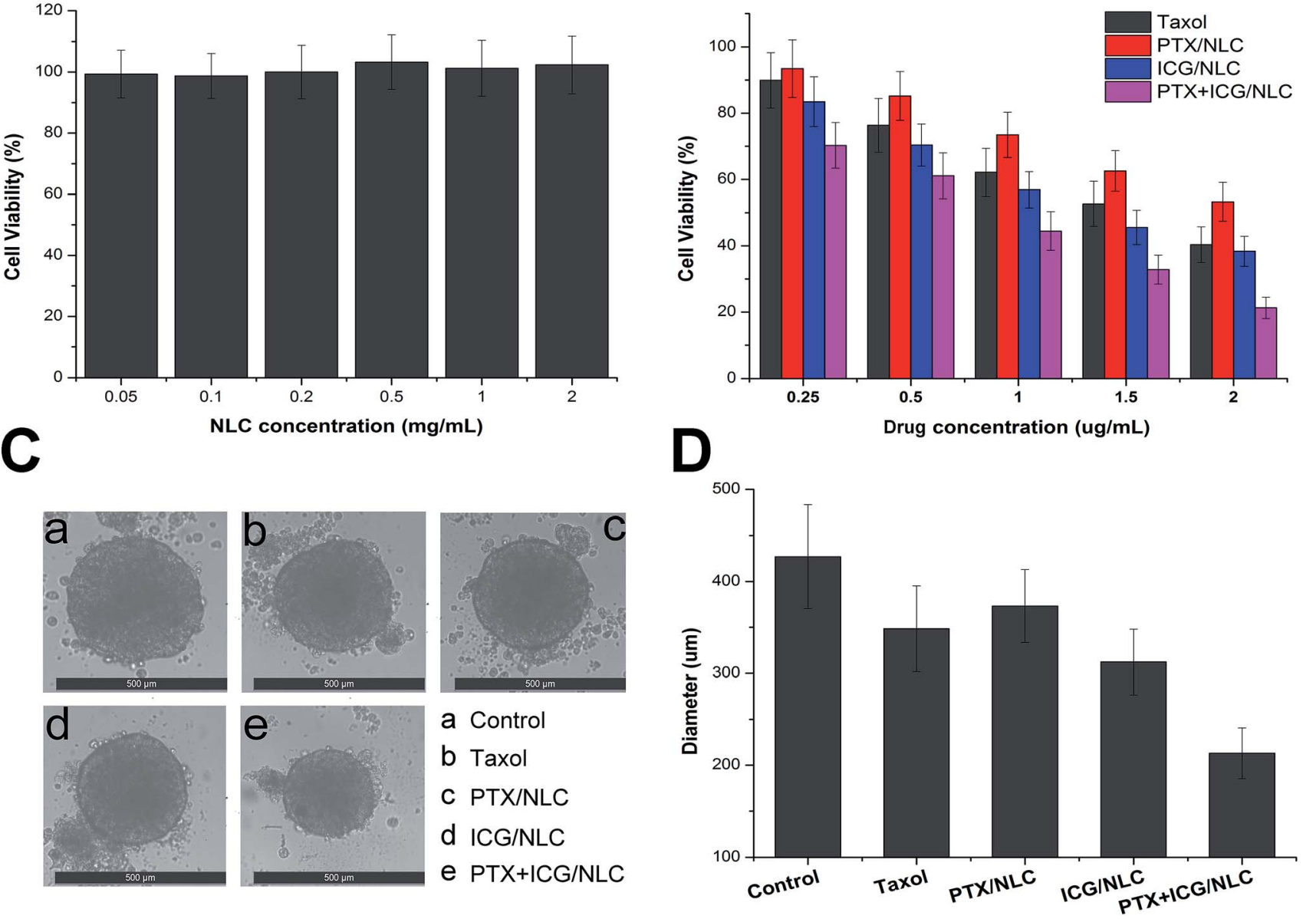

Fig. 7 (A) Cell viabilities of HepG2 cells incubated with free NLC at various concentrations for 48 h. (B) Cytotoxicity of Taxol, PTX/NLC, ICG/NLC and PTX + ICG/NLC with varying drug concentrations for $48 \mathrm{~h}$ and laser irritation in HepG2 cells. (C) Representative of Taxol, PTX/NLC, ICG/NLC and PTX + ICG/NLC treated 3D HepG2 spheroids at 4 days post incubation. (D) The diameter of Taxol, PTX/NLC, ICG/NLC and PTX + ICG/NLC treated 3D HepG2 spheroids at 4 days post incubation. Data were expressed as mean \pm S.D. $(n=3)$. 
which leads to significant fluorescence quenching and quick clearance might be responsible for such phenomenon.

This observation was consistent with previous reports that initial hepatic localization and eventual total clearance through the biliary tree with minimal acute renal involvement was the characteristic metabolic pathways of free ICG in vivo. ${ }^{22}$ In contrast, NLC possessed superior active tumor targeting ability, which is evidenced by the strong fluorescent signal at tumor site at $2 \mathrm{~h}$ post injection. The fluorescent signal in tumor increased over time and the intensity reached highest at $8 \mathrm{~h}$ postinjection. The comparative biodistributions of free ICG and NLC in major organs and tumors at $12 \mathrm{~h}$ after administration were shown in Fig. 6B, it was noticed that the fluorescent signal in tumor tissues of NLC treated mice was 28.48-fold of that in free ICG treated mice. This significant difference in ICG signal confirmed that NLC can effectively escort and deliver ICG to the wanted tumor sites when compared with free ICG.

\subsection{In vitro cytotoxicity assays}

In order to verify the synergetic effect of co-delivering PTX and ICG into one cancer cell, cytotoxicity of drug loaded NLC was examined by MTT assay. Firstly, the cytotoxicity of free NLC was tested to seek its toxic effect on cells. As shown in Fig. 7A, at all tested concentrations, even at the highest one of $2 \mathrm{mg} \mathrm{mL}^{-1}$, the NLC showed no or negligible toxic effect on cells, indicating that NLC is highly biocompatible. Besides, it could also inferred that the cytotoxicity effect of drug loaded NLC in the following experiments was all due to the therapeutic effect of either chemotherapy or PDT. As depicted in Fig. 7B, all the drugloaded formulations led to a dose-dependent decrease in cell viability after $48 \mathrm{~h}$ of treatment. On the other hand, it was also observed that both chemotherapy (PTX/NLC) and PDT (ICG/ NLC) could exert cytotoxicity on cancer cells to some extent. However, co-delivery of PTX and ICG that combined the advantages of both therapies could further enhance the cytotoxicity at the same drug concentrations. In order to further confirm this conclusion, we employed 3D HepG2 spheroids mimicking the solid tumors to test their anti-cancer effect. It was shown in Fig. 7C that after 4 days of co-incubation, the drug containing formulations can all reduce the diameter of HepG2 spheroids when compared with control group. It was worth mentioning that NLC co-delivering PTX and ICG exerted the most significant reduction on diameter of HepG2 spheroids according to results obtained in Fig. 7D. These results suggested that the co-delivery of PTX and ICG can synergistically exert their anti-cancer functions to achieve a better outcome. Thus, this biocompatible delivery vehicle significantly improves anti-cancer efficiency and promises to supply a novel platform for the conjunction of chemotherapy and PDT.

\section{Conclusions}

In conclusion, we successfully designed and formulated a highly biocompatible NLC that capable of co-delivering PTX and ICG for combined chemo and photodynamic therapy. It has been demonstrated in our study that NLC can effectively protect and deliver drugs to tumor cells for enhanced anti-cancer therapy. The biocompatible system not only significantly enhanced the stability of drugs, produced sufficient local ROS triggered by NIR laser irradiation, but also increased intracellular uptake of drugs and induced increased cytotoxicity in cancer cells via synergistic effects. Moreover, NLC demonstrated excellent tumor targetability in tumor-bearing mice. Therefore, tumor targeted co-delivery of PTX and ICG mediated by NLC could be a promising approach for further development as an effective strategy for cancer therapy.

\section{Acknowledgements}

We gratefully acknowledge the Program for Innovative Research Team in University of Jiangsu Province. We also appreciate the financial support from the National Natural Science Foundation of China (No. 21506096, 81603290 and 51603101), Natural Science Foundation of the Jiangsu Higher Education Institutions of China (No. 16KJB350001), National High Technology Research and Development Program of China (No. 2013AA020302) and National Key Research \& Development Plan of China (No. 2016YFC1201500).

\section{Notes and references}

1 C. Wang, S. Chen, Q. Yu, F. Hu and H. Yuan, J. Mater. Chem. $B$, 2017, 5, 2068-2073.

2 Y. Li, N. Chen, M. Palmisano and S. Zhou, Mol. Pharm., 2015, 12, 1308.

3 M. Kimura, M. Sano, M. Fujimori, H. Nakagomi, T. Negishi, Y. Yanagita and N. Sato, Anticancer Res., 2015, 28, 1239-1244.

4 X. Dong and R. J. Mumper, Nanomedicine, 2017, 5, 597.

5 B. Xu, X. Zhang, W. Huang, Y. Yang, Y. Ma, Z. Gu, T. Zhai and Y. Zhao, J. Mater. Chem. B, 2016, 4, 2776-2784.

6 B. Hou, B. Zhang, X. Gong, H. Wang, S. Wang, Z. Liao, X. Li, X. Zhang and J. Chang, J. Mater. Chem. B, 2015, 3, 3531-3540.

7 E.-H. Lee, J.-K. Kim, J.-S. Lim and S.-J. Lim, Colloids Surf., B, 2015, 136, 305-313.

8 X. G. Zhang, J. Miao, Y. Q. Dai, Y. Z. Du, H. Yuan and F. Q. Hu, Int. J. Pharm., 2008, 361, 239-244.

9 F. Q. Hu, S. P. Jiang, Y. Z. Du, H. Yuan, Y. Q. Ye and S. Zeng, Int. J. Pharm., 2006, 314, 83-89.

10 F. Q. Hu, S. P. Jiang, Y. Z. Du, H. Yuan, Y. Q. Ye and S. Zeng, Colloids Surf., B, 2005, 45, 167-173.

11 J. Miao, Y. Du, H. Yuan, X. Zhang, Q. Li, Y. Rao, M. Zhao and F. Hu, J. Nanopart. Res., 2015, 17, 10.

12 X. Y. Yang, Y. X. Li, M. Li, L. Zhang, L. X. Feng and N. Zhang, Cancer Lett., 2012, 334, 338-345.

13 X. Tang, Y. Cheng, S. Huang, A. Yuan, Y. Hu and J. Wu, Biochem. Biophys. Res. Commun., 2017, 487(3), 483-487.

14 C. Wang, M. Li, T. Yang, X. Ding, X. Bao, Y. Ding, H. Xiong, Y. Wu, W. Wang and J. Zhou, Mater. Sci. Eng., C, 2015, 56, 280-285.

15 X. Ding, W. Wang, Y. Wang, X. Bao, Y. Wang, C. Wang, J. Chen, F. Zhang and J. Zhou, Mol. Pharm., 2014, 11, 3307-3321. 
16 Y. Wang, C. Wang, Y. Ding, J. Li, M. Li, X. Liang, J. Zhou and W. Wang, Colloids Surf., B, 2016, 148, 533.

17 I. D'Angelo, C. Conte, A. Miro, F. Quaglia and F. Ungaro, Expert Opin. Drug Delivery, 2014, 11, 283-297.

18 K. Cai, Y. Hou, H. Yan, Z. Li, L. Zhong, Y. Shi, L. Min, W. Yang and L. Peng, Small, 2011, 7, 3026-3031.

19 A. Baeza, M. Colilla and M. Valletregí, Expert Opin. Drug Delivery, 2015, 12, 319.
20 C. Wang, X. Bao, X. Ding, Y. Ding, S. Abbad, Y. Wang, M. Li, Y. Su, W. Wang and J. Zhou, Polym. Chem., 2015, 6, 780-796.

21 B. Liu, C. Li, B. Xing, P. Yang and J. Lin, J. Mater. Chem. B, 2016, 4, 4884-4894.

22 T. Desmettre, J. M. Devoisselle and S. Mordon, Surv. Ophthalmol., 2000, 45, 15-27. 